\title{
Oral health and oral health behavior in young adults with caries disease
}

\author{
Jennie Hagman $\mathbb{D}^{1 凶}$, Ulla Wide ${ }^{1}$, Helene Werner ${ }^{1}$ and Magnus Hakeberg ${ }^{1}$
}

(c) The Author(s) 2021

OBJECTIVE: The aim of this study was to describe and analyze oral health, oral health behaviors, and oral health-related quality of life (OHRQoL) in relation to the level of caries disease among caries-active young adults.

MATERIAL AND METHODS: This study presents data from a sample of young adults $(n=135)$ with active caries disease who were enrolled in a clinical, randomized controlled trial. The independent variables of sociodemographics, oral health (gingivitis, plaque), oral health behaviors (such as toothbrushing, dental attendance, sugar-containing sweets and drinks), dental anxiety, self-rated oral health, and OHRQoL were collected. Multinomial logistic regression was used to simultaneously evaluate the associations between the independent variables and caries severity.

RESULTS: Multinominal logistic regression showed that poor OHRQoL and gingivitis were associated with caries severity in a gradient fashion in accordance with caries disease activity. Also, irregular dental care and frequent consumption of sugary soda were significantly associated with very high caries severity.

CONCLUSIONS: The risk factors related to caries severity among young adults were poor OHRQoL, gingivitis, consumption of sugary soda and irregular dental care attendance, indicating the need for a combination of different interventions specifically health behavior change. Furthermore, these findings may contribute to identifying high caries-risk individuals.

BDJ Open; https://doi.org/10.1038/s41405-021-00084-3

\section{INTRODUCTION}

Dental caries in permanent dentition is one of the world's most common diseases, affecting individuals throughout their lifetime. ${ }^{1}$ It may cause pain, discomfort, and anxiety and, if left untreated, lead to the spread of infection and tooth loss. This condition may not only affect an individual's ability to eat and speak properly but may also result in lost work and school hours and affect the individual's overall wellbeing. ${ }^{2}$ Dental caries is a multifactorial disease caused by both biological and behavioral factors, some of which (e.g., diet) are shared with other noncommunicable diseases (diabetes, heart disease, cancer). ${ }^{1}$

The caries prevalence has decreased substantially during the last decades, mainly in developed countries. In a repeated cross-sectional population survey among adults from 1983 to 2013, the caries prevalence dropped by $50 \%$, and for 35 -year-olds, the number of manifest caries lesions decreased from 2.0 to 0.9 over the 30-year time span. ${ }^{3}$ However, there are subgroups in populations that are affected by a high caries prevalence, which demonstrates the need for risk factor analysis as well as preventive and dental care interventions.

Several studies have been published concerning the associations between the effects of different risk factors (such as dental anxiety, oral health care habits, attitudes, socioeconomic status), over and above well-known etiological factors such as sugars and different types of bacteria with cariogenic properties, for the occurrence and distribution of oral diseases (i.e., dental caries). ${ }^{4-11}$ Little is known about the relationship between these risk factors and caries severity among young adults with a high disease burden. Identifying these key determinants of caries disease is important in the work to pursue more effective oral health promotion strategies in caries-active young adults, as well as finding high caries risk individuals in the clinic.

Furthermore, it is important to target young adults, as they are in the process of shaping their future adult health care habits. ${ }^{12}$ Establishing and maintaining positive oral health beliefs and confidence in the dental profession during early adulthood is crucial for the oral health outcome and oral health-related quality of life (OHRQoL), also later in life. ${ }^{13,14} \mathrm{~A}$ few reports have been published on dental caries and their association with OHRQoL in young adults. But the results are inconsistent, with some studies reporting an association, ${ }^{15-18}$ while others do not. ${ }^{19-21}$ Further studies of the relationship between caries disease and OHRQoL are therefore warranted.

According to Swedish law, the regions are obligated to provide dental care, free of charge, up to the age of 23 years, for all its inhabitants. Around $90 \%$ of all children, adolescents, and young adults attend the Public Dental Service clinics on a recall basis, based on both age and the risk of oral diseases. This makes the Public Dental Service (PDS) a suitable arena for research on oral health and oral health behavior among young adults.

The aim of this study was to describe and analyze oral health, oral health behaviors, and OHRQoL in relation to the level of caries disease among caries-active young adults.

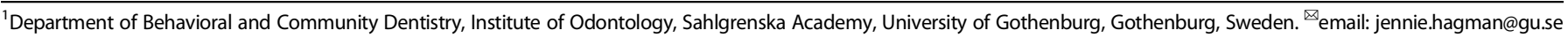




\section{MATERIAL AND METHODS}

\section{Design}

This study presents baseline data from a randomized controlled trial (RCT) (TRN ISRCTN15009620) that evaluated a psychological oral health intervention providing two cognitive behavior therapy sessions at dental clinics. $^{22}$ All participants in the RCT and a selection of baseline variables were included in the present study.

The Declaration of Helsinki protocols were followed, and the study was approved by The Regional Ethical Board in Gothenburg (reg. no. 840-12).

\section{Participants}

Recruitment of participants took place at two PDS clinics in Region Västra Götaland, Sweden, between 2013 and 2014. All young adults were screened for eligibility while attending their regular dental examination at their dentist or dental hygienist. Patients that met the inclusion criteria (18-25 years of age, and at least two manifest proximal dental caries lesions since the last dental examination) but not the exclusion criteria (psychiatric/neuropsychiatric diagnosis or not fluent in Swedish) were consecutively asked to participate. At the time of the recruitment of study participants, around ten dentists and six dental hygienists worked in each PDS clinic. Of 186 eligible individuals, 51 declined to participate, with the most common reasons being "not interested" $(n=24)$ and "lack of time" $(n=22)$. The remaining 135 individuals were included in the trial after they had given written informed consent. A more detailed description of the recruitment procedure has been published earlier. ${ }^{22}$

\section{Measurements}

Questions about sociodemographic variables, self-rated oral health, oral health behaviors, oral health-related quality of life, and dental anxiety were answered by the participants on a touch-screen computer.

Sociodemographic characteristics were measured with questions about sex, age, ethnicity (born in a Nordic country [Swedish-born, other Nordic country] or other countries); occupation (employed/student or unemployed); parents' country of birth (born in Nordic country [Swedish-born, other Nordic country] or other countries); parents' education (primary school, secondary school, university).

Self-rated oral health was measured with the question: "How do you rate your oral health?", with four response options (poor, fair, good, very good).

Oral health behaviors were captured with the questions "How often do you use a toothbrush?" and "How often do you use fluoridated toothpaste?", with six response options, dichotomized into $\geq$ twice a day (three times a day or more - twice a day), or $\leq$ once a day (once a day, several times a week, once a week, never or seldom); "How often do you use additional fluorides?" and "How often do you use dental floss?", with six response options, dichotomized into $\geq$ several times a week (three times a day or more, twice a day, once a day, several times a week), or $\leq$ once a week (once a week, never or seldom); "How often do you consume sugary sodas?" and "How often do you consume sweets?", with six response options, dichotomized into often (several times a day, once a day, several times a week), or seldom (once a week, seldom, never); "How often do you attend dental care?" was measured with six response options, dichotomized into $\geq$ once a year (twice a year, once a year), or $\leq$ once every second year (once every second year, less then every second year, only for acute dental need, never). "Do you smoke?" was measured with the response options "yes" or "no". The dichotomization of oral health behaviors was based on standard recommendations, ${ }^{23,24}$ while the variables sweets and sugary soda were dichotomized according to the midpoint of the scale used.

Oral health-related quality of life was assessed by the five-item version of the Oral Health Impact Profile (OHIP-5) questionnaire. ${ }^{25}$ It consists of five items addressing problems related to pain, oral function, oral psychosocial impact and appearance. Each item has five response alternatives (never (0), hardly ever, occasionally, fairly often, very often (4)), generating the sum score of $0-20$, with a higher score indicating poorer OHRQOL. The OHIP-5 was analyzed as a sum score and as dichotomized into good OHRQoL

Table 1. Sociodemographic characteristics, self-rated oral health, OHRQoL (measured by the OHIP-5), and dental anxiety (measured by the DAS) according to caries severity: moderate caries (2-3 lesions), high caries (4-6 lesions), very high caries ( $\geq 7$ lesions), and for the total sample.

\begin{tabular}{|c|c|c|c|c|c|}
\hline Variables $n$ (\%) & Moderate caries $(n=54)$ & High caries $(n=46)$ & Very high caries $(n=35)$ & $p$ value & Total $(n=135)$ \\
\hline Female & $26(48.1)$ & $20(43.5)$ & $18(51.4)$ & 0.829 & $64(47.4)$ \\
\hline Smoker & $13(24.1)$ & $19(41.3)$ & $15(42.9)$ & 0.052 & $47(34.8)$ \\
\hline Nordic-born & $44(81.5)$ & $37(80.4)$ & $22(62.9)$ & 0.058 & $103(76.3)$ \\
\hline Mother Nordic-born & $34(63.0)$ & $23(50.0)$ & $16(45.7)$ & 0.096 & $73(54.1)$ \\
\hline Father Nordic-born & $34(63.0)$ & $24(52.2)$ & $14(40.0)$ & 0.034 & $72(53.3)$ \\
\hline Employed/student & $42(77.8)$ & $39(84.8)$ & $23(65.7)$ & 0.266 & $104(77.0)$ \\
\hline \multicolumn{6}{|l|}{ Mother's education } \\
\hline Primary school & $12(22.2)$ & $14(30.4)$ & $11(31.4)$ & 0.108 & $37(27.4)$ \\
\hline Secondary school & $23(42.6)$ & $27(58.7)$ & $16(45.7)$ & & $66(48.9)$ \\
\hline University studies & $19(35.2)$ & $5(10.9)$ & $8(22.9)$ & & $32(23.7)$ \\
\hline \multicolumn{6}{|l|}{ Father's education } \\
\hline Primary school & $13(24.1)$ & $15(32.6)$ & $12(34.3)$ & 0.664 & $40(29.6)$ \\
\hline Secondary school & $32(59.3)$ & $20(43.5)$ & $16(45.7)$ & & $68(50.4)$ \\
\hline University studies & $9(16.7)$ & $11(23.9)$ & $7(20.0)$ & & $27(20.0)$ \\
\hline \multicolumn{6}{|l|}{ Self-rated oral health } \\
\hline Poor & $12(22.2)$ & $19(41.3)$ & $21(60.0)$ & 0.001 & $52(38.5)$ \\
\hline Fair & $30(55.6)$ & $22(47.8)$ & $10(28.6)$ & & $62(45.9)$ \\
\hline Good & $12(22.2)$ & $5(10.9)$ & $4(11.4)$ & & $21(15.6)$ \\
\hline Very good & $0(0.0)$ & $0(0.0)$ & $0(0.0)$ & & $0(0.0)$ \\
\hline OHIP-5, mean (SD) & $4.5(3.3)$ & $5.6(3.2)$ & $5.9(3.1)$ & 0.057 & $5.2(3.3)$ \\
\hline Poor OHRQoL ${ }^{a}$ & $33(61.1)$ & $37(80.4)$ & $29(82.9)$ & 0.016 & $99(73.3)$ \\
\hline DAS, mean (SD) & $7.7(4.0)$ & $7.8(3.7)$ & $9.8(4.6)$ & 0.049 & $8.3(4.1)$ \\
\hline
\end{tabular}

The Chi-square test was applied to categorical variables.

The Kruskal-Wallis test was used for continuous variables.

The Mann-Whitney test was applied for post-hoc analysis of continuous variables.

${ }^{\mathrm{a} O H I P-5}$ dichotomized. 
Table 2. Oral health behaviors and clinical parameters according to caries severity: moderate caries (2-3 lesions), high caries (4-6 lesions), very high caries ( $\geq 7$ lesions), and for the total sample.

\begin{tabular}{|c|c|c|c|c|c|}
\hline Variables $\boldsymbol{n}(\%)$ & $\begin{array}{l}\text { Moderate caries } \\
(n=54)\end{array}$ & $\begin{array}{l}\text { High caries } \\
(n=46)\end{array}$ & $\begin{array}{l}\text { Very high caries } \\
(n=35)\end{array}$ & $p$ value & Total $(n=135)$ \\
\hline Consuming sweets often & $22(40.7)$ & $16(34.8)$ & $13(37.1)$ & 0.687 & $51(37.8)$ \\
\hline Use of toothpaste $\geq$ twice a day & $35(64.8)$ & $25(54.3)$ & $24(68.6)$ & 0.856 & $84(62.2)$ \\
\hline Dental flossing $\geq$ several times a week & $21(38.9)$ & $14(30.4)$ & $16(45.7)$ & 0.630 & $51(37.8)$ \\
\hline Dental care attendance $\geq$ once a year & $48(88.9)$ & $42(91.3)$ & $24(68.6)$ & 0.019 & $114(84.4)$ \\
\hline Gingivitis, mean (SD) & $14.2(7.1)$ & $17.0(6.1)$ & $19.0(6.7)$ & 0.002 & $16.4(6.9)$ \\
\hline Plaque, mean (SD) & $5.9(6.2)$ & $8.8(6.2)$ & $8.5(6.6)$ & 0.022 & $7.6(6.4)$ \\
\hline
\end{tabular}

The Chi-square test was applied to categorical variables. The Kruskal-Wallis test was used for continuous variables. The Mann-Whitney test was applied for post-hoc analysis of continuous variables.

(scoring $\leq 1$ on all items) and poor OHRQoL (scoring $\geq 2$ on at least one item). ${ }^{17}$

Dental anxiety was assessed by the four-item Dental Anxiety Scale (DAS). ${ }^{26}$ The items are scored $1-5$, with sum scores of 4-20. A higher score indicates more dental anxiety.

Gingivitis was recorded at six index teeth $(16,21,24,44,41$, and 36$){ }^{27}$ at the buccal, mesial, distal, and lingual/palatinal surfaces. Gingivitis was present when bleeding was recorded on probing the gingival sulcus. ${ }^{28}$ In the present analysis, gingivitis is presented as the sum of surfaces diagnosed with gingivitis (range 0-24).

Plaque was recorded on the same index teeth and surfaces as for gingivitis and registered according to the Silness-Löe plaque index system, with each surface given a score between 0 and $3 .^{29}$ The plaque scores in the present analysis were dichotomized into "absence of visible plaque" (score $0-1$ ) or "presence of visible plaque" (score 2-3), and presented as the sum of surfaces with visible plaque (range 0-24).

Dental caries lesions were registered for all tooth surfaces by clinical and radiographic (bitewing radiographs) examination. Each surface was recorded as initial or manifest caries. ${ }^{30}$ The sum of manifest caries lesions was calculated for each participant. Additionally, the sum score for manifest lesions was trichotomized, based on the percentiles 33 and 67 , to create a moderate caries group (2-3 manifest lesions), a high caries group (4-6 manifest lesions), and a very high caries group ( $\geq 7$ manifest lesions), in order to evaluate associations in relation to other variables.

Interexaminer reliability was assessed for the diagnosis of manifest caries lesions on bite-wing radiographs $(n=31)$ with a kappa value of 0.82 , indicating very high agreement. ${ }^{31}$

\section{Statistical analyses}

Mean, medians (Md), standard deviations (SD), and frequencies were calculated for the descriptive analysis. For group comparisons, the Chisquare test was applied to categorical variables. The Kruskal-Wallis test was used to evaluate any difference between the three caries groups for continuous variables and the Mann-Whitney test was applied for post-hoc analysis. Multinomial logistic regression analyses were performed for the dependent outcome variable of caries, trichotomized into moderate, high, and very high caries groups. In the statistical analyses, the independent variables were divided into five different models based on similarities among the variables: (i) consumption of sugars, (ii) oral health behaviors, (iii) OHRQoL and dental anxiety, (iv) objective oral health, and (v) socioeconomic indicators. The Likelihood ratio test was used to evaluate the significance of each model and variables for the inclusion in the final model. The entry procedure was applied for each model. $P<0.05$ was chosen as level of significance.

\section{RESULTS}

\section{Sample characteristics}

Of the 135 individuals participating in the study, 64 were women and 71 men, mean age $20.6(S D=2.2)$. The participants had a mean number of $5.6(\mathrm{SD}=4.6)$ carious tooth surfaces and the proportion of tooth surfaces/gingival surfaces affected by plaque and gingivitis, were $32 \%$ and $68 \%$, respectively. Furthermore, the participants reported a mean OHIP-5 score of $5.2(\mathrm{SD}=3.3, \mathrm{Md}=5)$.

\section{Bivariate analyses}

Oral health behavior (i.e., consumption of sugary sodas, dental care attendance), sociodemographic characteristics (i.e., father's ethnicity), self-rated oral health, OHRQoL, dental anxiety, and clinical objective parameters (i.e., gingivitis, plaque levels) were found to differ significantly according to caries severity (Tables 1 and 2). In addition, the significant differences between caries groups showed a gradient feature with regard to caries severity.

Although no difference was shown between the three caries groups and the OHIP mean scores ( $p=0.057)$, a difference was found between the moderate and the very high caries groups $(p=0.030)$.

\section{Multivariable analysis}

In order to reveal associations between the independent variables and caries severity, five different statistical models that included theoretically important independent variables were analyzed. Based on these assumptions, four statistically significant models are shown in Table 3. The fifth model included socioeconomic indicators (sex, mother's education) and was found non-significant and therefore not included in Table 3 . The statistically significant variables in models $1-4$, respectively, were then included in a final model to reveal the association of each variable to caries severity. Thus, the variables consumption of sugary soda, dental care attendance, OHRQoL, and gingivitis were applied in the final model (Table 4). Specifically, belonging to the very high caries group rather than the moderate caries group was more likely if consuming sugary soda often, rather than less often, as well as reporting irregular dental care, rather than regular dental care. Moreover, reporting poor OHRQoL rather than good, as well as having high levels of gingivitis, were found to be associated with the high and very high caries groups compared with the moderate group.

\section{DISCUSSION}

This study showed a gradient association between caries severity and OHRQoL, sugar consumption, irregular dental care, and the amount of gingivitis among young adults with caries disease.

There are some limitations and strengths to this study that should be considered when interpreting the results. Firstly, the study lacks a healthy control group. Secondly, the cross-sectional 
Table 4. Multinomial logistic regression using caries group as the dependent variable with the moderate caries group as the reference group.

\begin{tabular}{|c|c|c|c|}
\hline & Final model & & \\
\hline & & OR & $95 \% \mathrm{Cl}$ \\
\hline \multirow[t]{7}{*}{ High caries group } & Sugary soda often & Ref. & \\
\hline & Sugary soda less often & 0.81 & $0.35-1.88$ \\
\hline & Irregular dental care & 0.83 & $0.21-3.23$ \\
\hline & Regular dental care & Ref. & \\
\hline & Good OHRQoL & 0.38 & $0.15-0.97$ \\
\hline & Poor OHRQoL & Ref. & \\
\hline & Gingivitis & 1.06 & $1.00-1.13$ \\
\hline \multirow{7}{*}{$\begin{array}{l}\text { Very high } \\
\text { caries group }\end{array}$} & Sugary soda often & Ref. & \\
\hline & Sugary soda less often & 0.35 & $0.12-0.98$ \\
\hline & Irregular dental care & 4.13 & $1.20-14.22$ \\
\hline & Regular dental care & Ref. & \\
\hline & Good OHRQoL & 0.37 & $0.12-1.17$ \\
\hline & Poor OHRQoL & Ref. & \\
\hline & Gingivitis & 1.11 & $1.03-1.20$ \\
\hline
\end{tabular}

OR Odds ratio, $95 \% \mathrm{Cl} 95 \%$ Confidence intervals, OHRQoL Oral healthrelated quality of life, Ref. Reference category.

Final model of included statistically significant independent variables.

study design implies that no causal links can be inferred. Thirdly, initial caries lesions were excluded from the analysis, thereby underestimating the actual caries prevalence in the sample and possibly also showing a weaker association between risk factors and the caries burden in the sample. Moreover, there are no data on oral health among the group of nonparticipants; hence, the severity of the caries burden in the sample may be underestimated. One strength of the study is that it consists of a fairly large clinical sample of young adults affected by a large number of manifest caries lesions. In addition, the sample comprised individuals attending general dental clinics at the PDS and not specialist/hospital clinics, which further strengthens the generalization perspective of the results. Furthermore, to our knowledge, there are no previous reports exploring oral health behavior, clinical status, OHRQoL and dental anxiety, and their associations with caries severity in a clinical sample of highly caries-active young adults.

In one study of Swedish 20-year olds, about $24 \%$ of the study sample had manifest caries lesions with a mean number of 0.5 surfaces with manifest caries lesion. ${ }^{32}$ In another Swedish epidemiological cross-sectional study, the number of cariesaffected surfaces (including both manifest and initial lesions) was 3.7 among 20-year-old study participants. ${ }^{33}$ In the present study, the mean number of dental surfaces with manifest caries lesion was 5.6, indicating that the study participants suffered from a high caries disease burden.

Previous research associated with caries experience in young adults lists risk factors such as socioeconomic and demographic factors, toothbrushing frequency, and dental anxiety. ${ }^{4-}$ 6,34-36 The present study did not find similar associations in the logistic regression analysis. In fact, there were four other factors that were significantly associated with higher levels of caries disease: gingivitis, OHRQoL, sugary soda consumption, and dental attendance behavior. Moreover, the present sample had a much higher proportion of negative oral health behaviors compared with the general population of adults, with respect to toothbrushing frequency and consumption of sugary sodas. ${ }^{37}$ For example, in the present sample, a high consumption of sugary sodas and less frequent toothbrushing 
were reported by $57.0 \%$ and $38.5 \%$, respectively. The corresponding figures for one Swedish study revealed $26 \%$ and $16 \%$, respectively, in a general population. ${ }^{37}$ Furthermore, in a population study of $19-20$-year-old Norwegians, $17.5 \%$ of the respondents reported that they consumed sugary sodas at least daily. ${ }^{38}$

Sociodemographic variables, such as migration background, parents born abroad, and parental educational level, are wellknown risk factors for dental caries; however, only father's ethnicity was found to be statistically significantly associated with the caries level in the present sample. Nevertheless, the association between these risk factors and dental caries were demonstrated in part, as the parents of the present study participants had a lower educational level in general and a greater proportion were born outside the Nordic countries, compared with the general population of region Västra Götaland at the time of the study. ${ }^{39}$

The vast majority of the study participants visited the dental clinic at least once a year; however, $15.6 \%$ visited only once every second year or even less frequent. In addition, the group of study participants that suffered from the highest burden of caries disease also had the greatest proportion of study participants that received dental care irregularly (31.4\%). Although the reason for this is not investigated in the present study, the results also demonstrated that this group reported the highest dental anxiety. There are several studies in the literature that have found that patients suffering from dental anxiety cancel/miss appointments more frequently and experience poorer oral health than a general population. ${ }^{40,41}$ This may be an explanation for the less frequent dental care attendance reported in this group. Another possible reason for not visiting the dental service every year may be misclassification by the dentists or dental hygienists regarding the caries risk among some of the study participants, resulting in longer re-call periods.

The level of dental anxiety in the present study is similar to the levels in previous reports about young adults, such as populationbased data for 26-year-old New Zealanders ${ }^{42}$ and 25-year-old Norwegians. ${ }^{43}$ However, the participants belonging to the very high caries group reported a statistically significantly higher mean score on the DAS compared with the moderate and high caries group, but the mean score did not reach the frequently used cutoff point for dental anxiety (score of $\geq 13$ ). ${ }^{44}$

To our knowledge, the OHIP-5 has not previously been used to measure OHRQoL in a young adult population affected by severe caries disease. However, the median value in the present sample $(M d=5)$ is clearly higher than the normative values of the Swedish population $(\mathrm{Md}=1)$, including the subpopulation of edentulous individuals and wearers of complete dentures $(\mathrm{Md}=$ 2). ${ }^{45}$ Even if the results from this study may not be directly comparable to those of other studies, due to factors such as different culture, age range, OHIP version, and study setting/ design, it is evident that the young adults in the present study suffer from poor OHRQoL. The present study revealed an association between the caries burden and poor OHRQoL, which is in line with previous findings by Lawrence et al., ${ }^{46}$ but in contrast to Flink et al. ${ }^{47}$ The proportion of the present sample reporting poor OHRQoL also seems to be very high (70\%), in contrast to findings in other studies (50\%). ${ }^{17,48}$

\section{CONCLUSIONS}

A higher burden of caries disease was associated with poorer oral health-related quality of life, more gingivitis, and higher consumption of sugar-containing sodas, and less frequent dental care attendance. It is obvious, based on these results, that despite the fact that all children in Sweden are offered full regular dental care free-of-charge up to young adulthood with a focus on prevention, young adults with severe caries disease still display a combination of different known risk factors with regard to health behaviors and perceived oral health-related quality of life. The findings may also contribute to identifying high caries-risk individuals. These individuals require special attention and care in the clinic. The dental profession may also need better tools in the clinic and collaboration with other professionals, such as dieticians and psychologists, in order to help individuals with severe caries disease.

\section{REFERENCES}

1. World Health Organization. Oral Health. https://www.who.int/news-room/factsheets/detail/oral-health (Accessed 2018).

2. Sheiham A. \& Conway D., Chestnutt I. 1.1 Impact of oral diseases and oral health inequalities In: Watt R. G., Listl S., Peres M., Heilmann A., (editors) Social Inequalities in Oral Health: From Evidence to Action. pp 4-5. UCL, (2015).

3. Edman, K., Ohrn, K., Nordstrom, B. \& Holmlund, A. Prevalence of dental caries and influencing factors, time trends over a 30 -year period in an adult population. Epidemiological studies between 1983 and 2013 in the county of Dalarna Sweden. Acta Odontol. Scand. 74, 385-392 (2016).

4. Julihn, A., Barr Agholme, M., Grindefjord, M. \& Modeer, T. Risk factors and risk indicators associated with high caries experience in Swedish 19-year-olds. Acta Odontol. Scand. 64, 267-273 (2006).

5. Roberts-Thomson, K. \& Stewart, J. F. Risk indicators of caries experience among young adults. Aust. Dent. J. 53, 122-127 (2008). quiz 186.

6. Jamieson, L. M., Mejia, G. C., Slade, G. D. \& Roberts-Thomson, K. F. Predictors of untreated dental decay among 15-34-year-old Australians. Community Dent. Oral. Epidemiol. 37, 27-34 (2009).

7. Sgan-Cohen, H. D., Katz, J., Horev, T., Dinte, A. \& Eldad, A. Trends in caries and associated variables among young Israeli adults over 5 decades. Community Dent. Oral. Epidemiol. 28, 234-240 (2000).

8. Kramer, A. C., Hakeberg, M., Petzold, M. \& Ostberg, A. L. Demographic factors and dental health of Swedish children and adolescents. Acta Odontol. Scand. 74 178-185 (2016).

9. Ericsson, J. S., Ostberg, A. L., Wennstrom, J. L. \& Abrahamsson, K. H. Oral healthrelated perceptions, attitudes, and behavior in relation to oral hygiene conditions in an adolescent population. Eur. J. Oral. Sci. 120, 335-341 (2012).

10. Ericsson, J. S. et al. Health investment behaviours and oral/gingival health condition, a cross-sectional study among Swedish 19-year olds. Acta Odontol. Scand. 74, 265-271 (2016).

11. Pitts, N. B. et al. Dental caries. Nat. Rev. Dis. Prim. 3, 17030 (2017)

12. Christie, D. \& Viner, R. Adolescent development. BMJ 330, 301-304 (2005)

13. Broadbent, J. M. et al. Oral health-related beliefs, behaviors, and outcomes through the life course. J. Dent. Res. 95, 808-813 (2016).

14. Broadbent, J. M., Thomson, W. M. \& Poulton, R. Oral health beliefs in adolescence and oral health in young adulthood. J. Dent. Res. 85, 339-343 (2006).

15. da Cunha, I. P. et al. Social vulnerability and factors associated with oral impact on daily performance among adolescents. Health Qual. life outcomes 15, 173 (2017).

16. Yamane-Takeuchi, M. et al. Associations among oral health-related quality of life, subjective symptoms, clinical status, and self-rated oral health in Japanese university students: a cross-sectional study. BMC Oral. Health 16, 127 (2016).

17. Drachev S. N., Brenn T. \& Trovik T. A. Oral Health-related quality of life in young adults: a survey of Russian undergraduate students. Int. J. Environ. Res. Public Health 15, 719 (2018)

18. Carvalho, J. C., Mestrinho, H. D., Stevens, S. \& van Wijk, A. J. Do oral health conditions adversely impact young adults? Caries Res. 49, 266-274 (2015).

19. Oscarson, N., Kallestal, C. \& Lindholm, L. A pilot study of the use of oral healthrelated quality of life measures as an outcome for analysing the impact of caries disease among Swedish 19-year-olds. Caries Res. 41, 85-92 (2007).

20. Choi, S. H., Kim, B. I., Cha, J. Y. \& Hwang, C. J. Impact of malocclusion and common oral diseases on oral health-related quality of life in young adults. Am. J. Orthod. Dentofac. Orthop. 147, 587-595 (2015).

21. Sun, L., Wong, H. M. \& McGrath, C. P. J. The factors that influence oral healthrelated quality of life in young adults. Health Qual. life outcomes 16, 187 (2018).

22. Wide, U., Hagman, J., Werner, H. \& Hakeberg, M. Can a brief psychological intervention improve oral health behaviour? A randomised controlled trial. BMC Oral. Health 18, 163 (2018).

23. Marinho V. C., Higgins J. P., Sheiham A., Logan S. Fluoride toothpastes for preventing dental caries in children and adolescents. Cochrane Datab. Syst. Rev. 2003:Cd002278.

24. Centers for Disease Control and Prevention. https://www.cdc.gov/healthywater/ hygiene/disease/dental_caries.html (Accessed 20 Jun 2021). 
25. John, M. T. et al. German short forms of the Oral Health Impact Profile. Community Dent. Oral. Epidemiol. 34, 277-288 (2006).

26. Corah, N. L. Development of a dental anxiety scale. J. Dent. Res. 48, 596 (1969).

27. Fleiss, J. L. et al. Representativeness of the "Ramfjord teeth" for epidemiologic studies of gingivitis and periodontitis. Community Dent. Oral. Epidemiol. 15, 221-224 (1987).

28. Muhlemann, H. R. \& Son, S. Gingival sulcus bleeding-a leading symptom in initial gingivitis. Helv. Odontol. Acta 15, 107-113 (1971).

29. Silness, J. \& Loe, H. Periodontal disease in pregnancy. II. Correlation between oral hygiene and periodontal condtion. Acta Odontol. Scand. 22, 121-135 (1964).

30. Pitts, N. B. \& Fyffe, H. E. The effect of varying diagnostic thresholds upon clinical caries data for a low prevalence group. J. Dent. Res. 67, 592-596 (1988).

31. Fayers, P. M. \& Machin, D. Quality of Life the Assessment, Analysis and Interpretation of Patient-Reported Outcomes. 2nd ed. (John Wiley \& Sons, Chichester, 2007).

32. Isaksson, H., Alm, A., Koch, G., Birkhed, D. \& Wendt, L. K. Caries prevalence in Swedish 20-year-olds in relation to their previous caries experience. Caries Res. 47, 234-242 (2013).

33. Norderyd, O. et al. Oral health of individuals aged 3-80 years in Jönköping, Sweden during 40 years (1973-2013). Il. Review of clinical and radiographic findings. Swed. Dent. J. 39, 69-86 (2015).

34. Ditmyer, M., Dounis, G., Mobley, C. \& Schwarz, E. A case-control study of determinants for high and low dental caries prevalence in Nevada youth. BMC Oral. Health 10, 24 (2010).

35. Skudutyte-Rysstad, R., Sandvik, L., Aleksejuniene, J. \& Eriksen, H. M. Dental health and disease determinants among 35-year-olds in Oslo, Norway. Acta Odontol. Scand. 67, 50-56 (2009).

36. Verlinden, D. A., Reijneveld, S. A., Lanting, C. I., van Wouwe, J. P. \& Schuller, A. A. Socio-economic inequality in oral health in childhood to young adulthood, despite full dental coverage. Eur. J. Oral Sci. 127, 248-253 (2019).

37. Norderyd, O. et al. Oral health of individuals aged 3-80 years in Jonkoping, Sweden, during 40 years (1973-52013). I. Review of findings on oral care habits and knowledge of oral health. Swed. Dent. J. 39, 57-68 (2015).

38. Asmyhr, $\varnothing$., Grytten, J. \& Holst, D. Occurrence of risk factors for dental erosion in the population of young adults in Norway. Community Dent. Oral. Epidemiol. 40, 425-431 (2012).

39. Statistics Sweden (SCB). http://www.statistikdatabasen.scb.se/pxweb/en/ssd/ (Accessed 20 Jun 2021).

40. Nermo, H., Willumsen, T. \& Johnsen, J. K. Prevalence of dental anxiety and associations with oral health, psychological distress, avoidance and anticipated pain in adolescence: a cross-sectional study based on the Troms $\varnothing$ study, Fit Futures. Acta Odontol. Scand. 77, 126-134 (2019).

41. Armfield, J. M., Slade, G. D. \& Spencer, A. J. Dental fear and adult oral health in Australia. Community Dent. Oral. Epidemiol. 37, 220-230 (2009).

42. Thomson, W. M., Locker, D. \& Poulton, R. Incidence of dental anxiety in young adults in relation to dental treatment experience. Community Dent. Oral. Epidemiol. 28, 289-294 (2000).

43. Astrom, A. N., Skaret, E. \& Haugejorden, O. Dental anxiety and dental attendance among 25-year-olds in Norway: time trends from 1997 to 2007. BMC Oral. Health 11, 10 (2011).

44. Corah, N. L., Gale, E. N. \& Illig, S. J. Assessment of a dental anxiety scale. J. Am. Dent. Assoc. 97, 816-819 (1978).

45. Larsson, P., John, M. T., Hakeberg, M., Nilner, K. \& List, T. General population norms of the Swedish short forms of oral health impact profile. J. Oral. Rehabil. 41, 275-281 (2014).

46. Lawrence, H. P., Thomson, W. M., Broadbent, J. M. \& Poulton, R. Oral healthrelated quality of life in a birth cohort of 32-year olds. Community Dent. Oral. Epidemiol. 36, 305-316 (2008).
47. Flink, H., Tegelberg, A., Arnetz, J. E. \& Birkhed, D. Self-reported oral and general health related to xerostomia, hyposalivation, and quality of life among caries active younger adults. Acta Odontol. Scand. 78, 229-235 (2020).

48. Lu, H.-X., Wong, M., Lo, E. \& McGrath, C. Oral health related quality of life among young adults. Appl. Res. Qual. Life. 10, 37-47 (2015).

\section{ACKNOWLEDGEMENTS}

The authors wish to thank the staff at the Public Dental Service clinics in Angered and Vänersborg, Region Västra Götaland, Sweden, for excellent collaboration during the study period, and especially research coordinators Camilla Svanberg and Erika Udvari Hargitai, as well as the heads of the participating clinics, Sigrid Nilsson and Mina Mehrpour. The study was supported by grants from The Health Care Subcommittee, Region Västra Götaland, Sweden.

\section{AUTHOR CONTRIBUTIONS}

M.H. and U.W. designed the study. M.H., U.W., H.W., and J.H. planned and performed the statistical analyses. J.H. drafted the manuscript. All authors participated in finalizing the manuscript. All authors gave their final approval and agreed to be accountable for all aspects of the work.

\section{FUNDING}

Open access funding provided by University of Gothenburg.

\section{COMPETING INTERESTS}

The authors declare no competing interests.

\section{ADDITIONAL INFORMATION}

Correspondence and requests for materials should be addressed to J.H.

Reprints and permission information is available at http://www.nature.com/ reprints

Publisher's note Springer Nature remains neutral with regard to jurisdictional claims in published maps and institutional affiliations.

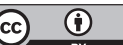

Open Access This article is licensed under a Creative Commons Attribution 4.0 International License, which permits use, sharing, adaptation, distribution and reproduction in any medium or format, as long as you give appropriate credit to the original author(s) and the source, provide a link to the Creative Commons license, and indicate if changes were made. The images or other third party material in this article are included in the article's Creative Commons license, unless indicated otherwise in a credit line to the material. If material is not included in the article's Creative Commons license and your intended use is not permitted by statutory regulation or exceeds the permitted use, you will need to obtain permission directly from the copyright holder. To view a copy of this license, visit http://creativecommons. org/licenses/by/4.0/.

(c) The Author(s) 2021 